\title{
Characterizations of Hemirings Based on Probability Spaces
}

\author{
Bin Yu and Jianming Zhan \\ Department of Mathematics, Hubei University for Nationalities, Enshi, Hubei 445000, China \\ Correspondence should be addressed to Jianming Zhan; zhanjianming@hotmail.com
}

Received 24 March 2013; Accepted 8 May 2013

Academic Editor: Qiankun Song

Copyright (C) 2013 B. Yu and J. Zhan. This is an open access article distributed under the Creative Commons Attribution License, which permits unrestricted use, distribution, and reproduction in any medium, provided the original work is properly cited.

The notion of falling fuzzy $h$-ideals of a hemiring is introduced on the basis of the theory of falling shadows and fuzzy sets. Then the relations between fuzzy $h$-ideals and falling fuzzy $h$-ideals are described. In particular, by means of falling fuzzy $h$-ideals, the characterizations of $h$-hemiregular hemirings are investigated based on independent (prefect positive correlation) probability spaces.

\section{Introduction}

Starting from a unified treatment of uncertainty by combining probability and fuzzy set theory [1], Goodman [2] put forward the equivalence between a fuzzy set and a class of random sets. Falling shadow representation theory was established based on the collection of Wang and Sanchez [3], which is directly related to the concept of probabilistic fuzzy set membership function. The theory shows the selection methods related to the joint degrees distributions. It provides a reasonable and convenient approach for the theoretical development and the practical applications of fuzzy sets and fuzzy logics. Utilizing the theory of falling shadows, in particular, Tan et al. $[4,5]$ established a theoretical approach to define a fuzzy inference relation and fuzzy set operations based on the theory of falling shadows. Yuan and Lee [6] considered a fuzzy subgroup (subring, ideal) as the falling shadow of a cloud of the subgroups (subrings, ideals). Jun and Kang [7] proposed a theoretical approach for BCK algebras.

A semiring plays an important role in studying matrices and determinants. Many aspects of the theory of matrices and determinants over semiring have been studied by Beasley and Pullman [8] and Ghosh [9]. The ideals in semiring are useful for many purposes, but they do not coincide with the usual ring ideals if $S$ is ring in general. Their use is thus somewhat limited in terms of obtaining analogues of ring theorems for semirings. In fact, many results in ring apparently have no analogues in hemirings using only ideals. LaTorre [10] investigated $h$-ideals and $k$-ideals in hemirings in an effort to obtain analogues of familiar ring theorems.
The fuzzy theory in semirings and hemirings has been discussed by many researchers (see [11-16]). The concept of $h$-hemiregular hemirings has been introduced by Zhan and Dudek [17] to generalize the regularity in hemirings. Further, some characterizations of $h$-semisimple and $h$-intrahemiregular hemirings were investigated by Yin et al. $[18,19]$. It is pointed out that some generalized fuzzy $h$-ideals of hemirings were investigated by Ma et al.; for example, see [18-25].

Recently, some properties of falling fuzzy ideals of hemirings have also been investigated by Yu and Zhan [26]. As a continuation of our previous investigation of falling fuzzy ideals of hemirings, the present paper is organized as follows. In Section 2, we recall the concepts and properties of hemirings, fuzzy sets, and falling shadows. In Section 3, we introduce the concept of falling fuzzy $h$-ideals and investigated some related properties. Finally, we investigate characterizations of $h$-hemiregular hemirings based on independent (prefect positive correlation) probability spaces in Section 4 .

\section{Preliminaries}

A semiring is an algebraic system $(S,+, \cdot)$ consisting of a nonempty set $S$ together with two binary operations on $S$ called addition and multiplication (denoted in the usual manner) such that $(S,+)$ and $(S, \cdot)$ are semigroups and the following distributive laws:

$$
a(b+c)=a b+a c, \quad(a+b) c=a c+b c
$$

are satisfied for all $a, b, c \in S$. 
By zero of a semiring $(S,+, \cdot)$ we mean an element $0 \in S$ such that $0 \cdot x=x \cdot 0=0$ and $0+x=x+0=0$ for all $x \in S$. A semiring with zero and a commutative semigroup $(S,+)$ are called a hemiring. For the sake of simplicity, we shall write $a b$ for $a \cdot b(a, b \in S)$.

A subset $A$ in a hemiring $S$ is called a left (right) ideal of $S$ if $A$ is closed under addition and $S A \subseteq A(A S \subseteq A)$. Further, $A$ is called an ideal of $S$ if it is both a left ideal and a right ideal of $S$. A left $h$-ideal of hemiring $S$ is defined to be a left ideal $A$ of $S$, such that, for all $x, z \in S$, and, for all $a, b \in A, x+a+z=b+z \rightarrow x \in A$.

The $h$-closure $\bar{A}$ of $A$ in a hemiring $S$ is defined as: $\bar{A}=$ $\left\{x \in S \mid x+a_{1}+z=a_{2}+z\right.$ for some $\left.a_{1}, a_{2} \in A, z \in S\right\}$.

Definition 1 (see [25]). A fuzzy set $\mu$ of a hemiring $S$ is called a fuzzy left (right) $h$-ideal if, for all $x, y, z, a, b \in S$, we have

$$
\begin{aligned}
& \left(F_{1}\right) \mu(x+y) \geq \min \{\mu(x), \mu(y)\} \\
& \left(F_{2}\right) \mu(x y) \geq \mu(y)(\mu(x y) \geq \mu(x)) \\
& \left(F_{3}\right) x+a+z=b+z \rightarrow \mu(x) \geq \min \{\mu(a), \mu(b)\} .
\end{aligned}
$$

Further, $\mu$ is called a fuzzy $h$-ideal of $S$ if it is both a fuzzy left $h$-ideal and a fuzzy right $h$-ideal of $S$.

Note that if $\mu$ is a fuzzy $h$-ideal, then $\mu(0) \geq \mu(x)$ for all $x \in S$.

For any $A \subseteq S$, we denote the characteristic function of $A$ by $\chi_{A}$.

Theorem 2 (see [17]). A fuzzy set $\mu$ of $S$ is a fuzzy h-ideal of $S$ if and only if the nonempty subset $\mu_{t}$ is an $h$-ideal of $S$ for all $t \in[0,1]$.

It is well known that ideals theory plays a fundamental role in the development of hemirings. Throughout this paper, $S$ is a hemiring.

We now display the basic theory on falling shadows. We refer the reader to the papers [2-5] for further information regarding falling shadows. Given a universe of discourse $U$, let $\mathscr{P}(\mathcal{U})$ denote the power set of $U$. For each $u \in U$, let

$$
\dot{u}=\{E \mid u \in E, E \subseteq U\},
$$

and, for each $E \in \mathscr{P}(U)$, let

$$
\dot{E}=\{\dot{u} \mid u \in E\} .
$$

An ordered pair $(\mathscr{P}(U), \mathscr{B})$ is said to be a hypermeasurable structure on $U$ if $\mathscr{B}$ is a $\sigma$-field in $\mathscr{P}$ and $\dot{U} \subseteq \mathscr{B}$. Given a probability space $(\Omega, \mathscr{A}, P)$ and a hypermeasurable structure $(\mathscr{P}(U), \mathscr{B})$ on $U$, a random set on $U$ is defined to be a mapping $\xi: \Omega \rightarrow \mathscr{P}(U)$, which is $\mathscr{A}-\mathscr{B}$ measurable, that is,

$$
\xi^{-1}(C)=\{\omega \mid \omega \in \Omega, \xi(\omega) \in C\} \in \mathscr{A}, \quad \forall C \in \mathscr{B} .
$$

Suppose that $\xi$ is a random set on $U$. Let

$$
\widetilde{H}(u)=P(\omega \mid u \in \xi(\omega)), \quad \text { for each } u \in U .
$$

Then $\widetilde{H}$ is a kind of fuzzy set in $U$. We call $\widetilde{H}$ a falling shadow of the random set $\xi$, and $\xi$ is called a cloud of $\widetilde{H}$.

For example, $(\Omega, \mathscr{A}, P)=([0,1], \mathscr{A}, m)$, where $\mathscr{A}$ is a Borel field on [0,1] and $m$ is the usual Lebesgue measure. Let $\widetilde{H}$ be a fuzzy set in $U$ and $\widetilde{H}_{t}=\{u \in U \mid \widetilde{H}(u) \geq t\}$ be a $t$-cut of $\widetilde{H}$. Then

$$
\xi:[0,1] \longrightarrow \mathscr{P}(U), \quad t \longmapsto \widetilde{H}_{t}
$$

is a random set and $\xi$ is a cloud of $\widetilde{H}$. We shall call $\xi$ defined above the cut cloud of $\widetilde{H}$ (see [2]).

\section{Falling Fuzzy $h$-Ideals}

In this section, we will introduce the notion of falling fuzzy $h$-ideals of a hemiring. The relations between fuzzy $h$-ideals and falling fuzzy $h$-ideals are provided.

Definition 3 (see [26]). Let $(\Omega, \mathscr{A}, \mathscr{P})$ be a probability space, and let $\xi: \Omega \rightarrow \mathscr{P}(\delta)$ be a random set. If $\xi(\omega)$ is a left (right) ideal of $S$ for any $\omega \in \Omega$, then the falling shadow of the random set $\xi$, that is, $\widetilde{H}(u)=P(\omega \mid u \in \xi(\omega))$, is called a falling fuzzy left (right) ideal of $S$. Further, $\widetilde{H}(u)$ is called a falling fuzzy ideal of $S$ if it is both a falling fuzzy left ideal and a falling fuzzy right ideal of $S$.

Let $(\Omega, \mathscr{A}, P)$ be a probability space and $F(S)=\{f \mid f$ : $\Omega \rightarrow S\}$, where $S$ is a hemiring.

Define two operations $\oplus$ and $\odot$ on $F(S)$ by

$$
\begin{aligned}
& (f \oplus g)(\omega)=f(\omega)+g(\omega), \\
& (f \odot g)(\omega)=f(\omega) \cdot g(\omega),
\end{aligned}
$$

for all $\omega \in \Omega, f, g \in F(S)$.

Let $\theta \in F(S)$ be defined by $\theta(\omega)=0$, for all $\omega \in \Omega$. Then we can check that $(F(S), \oplus, \odot, \theta)$ is a hemiring.

For any subset $A$ of $S$ and $f \in F(S)$, let $A_{f}=\{\omega \in \Omega \mid$ $f(\omega) \in A\}$,

$$
\begin{gathered}
\xi: \Omega \longrightarrow \mathscr{P}(F(S)), \\
\omega \longmapsto\{f \in F(S) \mid f(\omega) \in A\},
\end{gathered}
$$

and then $A_{f} \in \mathscr{A}$.

Definition 4 . Let $(\Omega, \mathscr{A}, \mathscr{P})$ be a probability space, and let $\xi$ : $\Omega \rightarrow \mathscr{P}(\mathcal{S})$ be a random set. If $\xi(\omega)$ is an $h$-ideal of $S$ for any $\omega \in \Omega$, then the falling shadow of the random set $\xi$, that is, $\widetilde{H}(u)=P(\omega \mid u \in \xi(\omega))$, is called a falling fuzzy $h$-ideal of $S$.

Proposition 5. If $A$ is an $h$-ideal of $S$, then $\xi(\omega)=\{f \in F(S) \mid$ $f(\omega) \in A\}$ is an $h$-ideal of $F(S)$.

Proof. Assume that $A$ is an $h$-ideal of $S$ and $\omega \in \Omega$. Let $f, g \in$ $F(S)$ be such that $f, g \in \xi(\omega)$, and then $f(\omega), g(\omega) \in A$. Since $A$ is an $h$-ideal of $S$, then $f(\omega)+g(\omega) \in A$. Thus, $(f \oplus g)(\omega)=$ $f(\omega)+g(\omega) \in A$, and so $f \oplus g \in \xi(\omega)$. Let $f \in \xi(\omega)$ and $t \in F(S)$, and then $f(\omega) \in A$. Since $A$ is an $h$-ideal of $S$, then $(t \odot f)(\omega)=t(\omega) \cdot f(\omega) \in A$, and so $t \odot f \in \xi(\omega)$, that is, $F(S) \odot \xi(\omega) \in \xi(\omega)$. Similarly, we can prove $\xi(\omega) \odot F(S) \in \xi(\omega)$. 
Let $f, g \in \xi(\omega)$ and $t, h \in F(S)$, and then $f(\omega), g(\omega) \in A$. Hence $(t \oplus f \oplus h)(\omega)=t(\omega)+f(\omega)+h(\omega)=g(\omega)+h(\omega)=$ $(g \oplus h)(\omega)$. Since $A$ is an $h$-ideal of $S$, we have $t(\omega) \in A$, that is, $t \in \xi(\omega)$. Hence, $\xi(\omega)$ is an $h$-ideal of $F(S)$.

From the above proposition, we know that $\widetilde{H}$ is a falling fuzzy ideal of $F(S)$, where $\widetilde{H}(f)=P(\omega \mid f(\omega) \in A\}$. In fact, since

$$
\begin{aligned}
\xi^{-1}(f) & =\{\omega \in \Omega \mid f \in \xi(\omega)\} \\
& =\{\omega \in \Omega \mid f(\omega) \in A\} \\
& =A_{f} \in \mathscr{A},
\end{aligned}
$$

we see that $\xi$ is a random set on $F(S)$. By Proposition 5, we know that $\widetilde{H}$ is a falling fuzzy $h$-ideal of $S$.

Example 6. (1) Let $S=\{0,1,2,3\}$ be a set with an addition operation and a multiplication operation as follows:

$$
\begin{aligned}
& \begin{array}{l|llll}
+ & 0 & 1 & 2 & 3 \\
\hline 0 & 0 & 1 & 2 & 3
\end{array} \quad \begin{array}{c|cccc}
0 & 0 & 1 & 2 & 3 \\
\hline & 1 & 1 & 2 & 3
\end{array}
\end{aligned}
$$

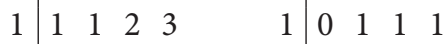

$$
\begin{aligned}
& \begin{array}{llllllllllll}
2 & 2 & 2 & 2 & 3 & & & 2 & 0 & 1 & 1 & 1
\end{array} \\
& \begin{array}{l|llllll|llll}
3 & 3 & 3 & 3 & 2 & & 3 & 0 & 1 & 1 & 1
\end{array}
\end{aligned}
$$

Then $(S,+, \cdot)$ is a hemiring [23].

Let $(\Omega, \mathscr{A}, P)=([0,1], \mathscr{A}, m)$ and $\xi:[0,1] \rightarrow \mathscr{P}(S)$ be defined by

$$
\xi(t)= \begin{cases}\{0\}, & \text { if } t \in[0,0.3) \\ \{0,1\}, & \text { if } t \in[0.3,0.5) \\ \{0,1,2\}, & \text { if } t \in[0.5,0.9) \\ S, & \text { if } t \in[0.9,1]\end{cases}
$$

Then $\xi(t)$ is an $h$-ideal of $S$ for all $t \in[0,1]$. Hence $\widetilde{H}=$ $P(t \mid x \in \xi(t))$ is a falling fuzzy $h$-ideal of $S$. bles:

(2) The set $S=\{0,1, a, b, c\}$ with the following Cayley ta-

$$
\begin{aligned}
& \begin{array}{l|lllll}
+ & 0 & 1 & a & b & c \\
\hline 0 & 0 & 1 & a & b & c
\end{array} \quad+\cdot \begin{array}{llllll}
0 & 1 & a & b & c \\
\hline 0 & 0 & 0 & 0 & 0 & 0
\end{array} \\
& \begin{array}{lllllll|lllll}
1 & 1 & b & 1 & a & 1 & 1 & 0 & 1 & a & b & c
\end{array} \\
& \begin{array}{llllllllllll}
a & a & 1 & a & b & a & a & 0 & a & a & a & c
\end{array} \\
& \begin{array}{llllllll|lllll}
b & b & a & b & 1 & b & b & 0 & b & a & 1 & c
\end{array}
\end{aligned}
$$

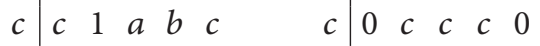

Then $(S,+, \cdot)$ is a hemiring.

Let $(\Omega, \mathscr{A}, P)=([0,1], \mathscr{A}, m)$ and $\xi:[0,1] \rightarrow \mathscr{P}(S)$ be defined by

$$
\xi(t)= \begin{cases}\{0\}, & \text { if } t \in[0,0.3) \\ \{0, a, c\}, & \text { if } t \in[0.3,0.5) \\ S, & \text { if } t \in[0.5,1]\end{cases}
$$

Then $\xi(t)$ is an $h$-ideal of $S$ for all $t \in[0,1]$. Hence $\widetilde{H}=$ $P(t \mid x \in \xi(t))$ is a falling fuzzy $h$-ideal of $S$.

Theorem 7. Every fuzzy $h$-ideal of $S$ is a falling fuzzy $h$-ideal of $S$.
Proof. Consider the probability space $(\Omega, \mathscr{A}, P)=([0,1], \mathscr{A}$, $m)$, where $\mathscr{A}$ is a Bored field on $[0,1]$, and $m$ is the usual Lebesgue measure. Let $\mu$ be a fuzzy $h$-ideal of $S$, and then $\mu_{t}$ is an $h$-ideal of $S$ for all $t \in[0,1]$. Let $\xi:[0,1] \rightarrow \mathscr{P}(S)$ be a random set and $\xi(t)=\mu_{t}$ for every $t \in[0,1]$. Then $\mu$ is a falling fuzzy $h$-ideal of $S$.

Remark 8. The following example shows that the converse of Theorem 7 is not valid.

Example 9. Let $S=\{0, a, b, c\}$ be a set with an addition operation and a multiplication operation as follows:

$$
\begin{aligned}
& \begin{array}{l|llll}
+ & 0 & a & b & c \\
\hline 0 & 0 & a & b & c
\end{array} \quad \cdot \begin{array}{l|llll}
0 & a & b & c \\
\hline & a & 0 & c & b
\end{array} \\
& \begin{array}{lllllllllll}
a & a & 0 & c & b & a & 0 & 0 & 0 & 0
\end{array}
\end{aligned}
$$

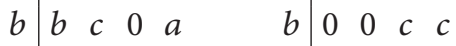

$$
\begin{aligned}
& \begin{array}{lllllllllll}
c & c & b & a & 0 & & c & 0 & 0 & c & c
\end{array}
\end{aligned}
$$

Then $(S,+, \cdot)$ is a hemiring.

Let $(\Omega, \mathscr{A}, P)=([0,1], \mathscr{A}, m)$ and $\xi:[0,1] \rightarrow \mathscr{P}(S)$ be defined by

$$
\xi(t)= \begin{cases}\{0\}, & \text { if } t \in[0,0.3) \\ \{0, a\}, & \text { if } t \in[0.3,0.5) \\ \{0, c\}, & \text { if } t \in[0.5,0.9) \\ S, & \text { if } t \in[0.9,1]\end{cases}
$$

Then $\xi(t)$ is an $h$-ideal of $S$ for all $t \in[0,1]$. Hence $\widetilde{H}=P(t \mid$ $x \in \xi(t))$ is a falling fuzzy $h$-ideal of $S$, and it is represented as follows:

$$
\widetilde{H}(x)= \begin{cases}1, & \text { if } x=0 \\ 0.3, & \text { if } x=a \\ 0.5, & \text { if } x=c \\ 0.1, & \text { if } x=b\end{cases}
$$

Then

$$
\widetilde{H}_{t}= \begin{cases}\{0\}, & \text { if } t \in(0.5,1] \\ \{0, c\}, & \text { if } t \in(0.3,0.5] \\ \{0, a, c\}, & \text { if } t \in(0.1,0.3] \\ S, & \text { if } t \in[0,0.1]\end{cases}
$$

If $t \in(0.1,0.3]$, then $\widetilde{H}_{t}=\{0, a, c\}$ is not an $h$-ideal of $S$ since $a+c=b \nsubseteq\{0, a, c\}$. Thus, it follows from Theorem 2 that $\widetilde{H}$ is not a fuzzy $h$-ideal of $S$.

Let $(\Omega, \mathscr{A}, P)$ be a probability space and a falling shadow of a random set $\xi: \Omega \rightarrow \mathscr{P}(S)$. For any $x \in S$, let $\Omega(x ; \xi)=$ $\{\omega \in \Omega \mid x \in \xi(\omega)\}$. Then $\Omega(x ; \xi) \in \mathscr{A}$.

Theorem 10. If a falling shadow $\widetilde{H}$ of a random set $\xi: \Omega \rightarrow$ $\mathscr{P}(S)$ is a falling fuzzy left (right) $h$-ideal of $S$, then, for all $x, y, a, b, z \in S$, we have
(1) $\Omega(x ; \xi) \cap \Omega(y ; \xi) \subseteq \Omega(x+y ; \xi)$;
(2) $\Omega(y ; \xi) \subseteq \Omega(x y ; \xi)(\Omega(x ; \xi) \subseteq \Omega(x y ; \xi))$;
(3) $x+a+z=b+z \Rightarrow \Omega(a ; \xi) \cap \Omega(b ; \xi) \subseteq \Omega(x ; \xi)$. 
Proof. (1) Let $\omega \in \Omega(x ; \xi) \cap \Omega(y ; \xi)$, then $x, y \in \xi(\omega)$. Since $\xi(\omega)$ is a left (right) $h$-ideal of $S$ by Definition 4 , then $x+y \in$ $\xi(\omega)$, and so $\omega \in \Omega(x+y ; \xi)$. This implies that $\Omega(x ; \xi) \cap$ $\Omega(y ; \xi) \subseteq \Omega(x+y ; \xi)$.

(2) Let $\omega \in \Omega(y ; \xi)$, and then $y \in \xi(\omega)$. Since $\xi(\omega)$ is a left $h$-ideal of $S$ by Definition 4 , then $x y \in \xi(\omega)$, and so $\omega \in \Omega(x y ; \xi)$. This implies that $\Omega(y ; \xi) \subseteq \Omega(x y ; \xi)$. Similarly, we can show that $\Omega(x ; \xi) \subseteq \Omega(x y ; \xi)$.

(3) Let $\omega \in \Omega(a ; \xi) \cap \Omega(b ; \xi)$ and $x, z \in S$, and then $a, b \in$ $\xi(\omega)$. Since $\xi(\omega)$ is a left (right) $h$-ideal of $S$ by Definition 4 , then $x \in \xi(\omega)$, and so $\omega \in \Omega(x ; \xi)$. This implies that $\Omega(a ; \xi) \cap$ $\Omega(b ; \xi) \subseteq \Omega(x ; \xi)$. This completes the proof.

Theorem 11. Let $\widetilde{H}$ be a falling fuzzy $h$-ideal of $S$, and then $\widetilde{H}(x y) \geq \max \{\widetilde{H}(x), \widetilde{H}(y)\}$.

Proof. Since $\Omega(x y ; \xi) \supseteq \Omega(x ; \xi) \cap \Omega(y ; \xi)$, it follows that

$$
\begin{aligned}
\widetilde{H}(x y)= & P(\omega \mid x y \in \xi(\omega)) \\
\geq & P((\omega \mid x \in \xi(\omega)) \cap(\omega \mid y \in \xi(\omega))) \\
\geq & P(\omega \mid x \in \xi(\omega))+P(\omega \mid y \in \xi(\omega)) \\
& -P((\omega \mid x \in \xi(\omega)) \cap(\omega \mid y \in \xi(\omega))) \\
\geq & \max \{P(\omega \mid x \in \xi(\omega)), P(\omega \mid y \in \xi(\omega))\} \\
= & \max \{\widetilde{H}(x), \widetilde{H}(y)\} .
\end{aligned}
$$

\section{Characterizations of $h$-Hemiregular Hemirings}

The concept of $h$-hemiregularity of a hemiring was first introduced by Zhan and Dudek [17] as a generalization of the concept of regularity of a ring.

Definition 12 (see [25]). A hemiring $S$ is said to be $h$ hemiregular if, for each $a \in S$, there exist $x_{1}, x_{2}, z \in S$ such that $a+a x_{1} a+z=a x_{2} a+z$.

Lemma 13 (see [17]). If $A$ and $B$ are, respectively, $a$ right $h$ ideal and a left $h$-ideal of a hemiring $S$, then $\overline{A B} \subseteq A \cap B$.

Lemma 14 (see [17]). A hemiring $S$ is hemiregular if and only if, for any right $h$-ideal $A$ and for any left $h$-ideal $B, \overline{A B}=A \cap B$.

In the following sections, we divide the results into two parts. In Sections 4.1 and 4.2, we describe the characterizations of $h$-hemiregular hemirings based on prefect positive correlation and independent probability spaces via falling fuzzy $h$-ideals, respectively.

4.1. Prefect Positive Correlation Probability Spaces. In this subsection, we describe the characterizations of $h$-hemiregular hemirings based on prefect positive correlation probability spaces via falling fuzzy $h$-ideals.
Definition 15. The probability space $\Omega$ is called prefect positive correlation if $\Omega(x ; \xi) \subseteq \Omega(y ; \xi)$ or $\Omega(y ; \xi) \subseteq \Omega(x ; \xi)$ for all $x, y \in S$.

Definition 16. Let $\Omega$ be a prefect positive correlation probability space and let $\widetilde{H}_{1}$ and $\widetilde{H}_{2}$ be falling fuzzy $h$-ideals of $S$. Then the $P$ product of $\widetilde{H}_{1}$ and $\widetilde{H}_{2}$ is defined by

$$
\begin{aligned}
& \left(\widetilde{H}_{1} \odot_{p} \widetilde{H}_{2}\right)(x) \\
& =\sup _{x+\sum_{i=1}^{m} a_{i} b_{i}+z=\sum_{j=1}^{n} a_{j}^{\prime} b_{j}^{\prime}+z}\left(\operatorname { m i n } \left\{\widetilde{H}_{1}\left(a_{i}\right), \widetilde{H}_{1}\left(a_{j}^{\prime}\right), \widetilde{H}_{2}\left(b_{i}\right),\right.\right. \\
& \widetilde{H}_{2}\left(b_{j}^{\prime}\right) \mid i=1, \ldots, m, \\
& j=1, \ldots n\})
\end{aligned}
$$

and $\left(\widetilde{H}_{1} \odot_{p} \widetilde{H}_{2}\right)(x)=0$ if $x$ cannot be expressed as $x+$ $\sum_{i=1}^{m} a_{i} b_{i}+z=\sum_{j=1}^{n} a_{j}^{\prime} b_{j}^{\prime}+z$.

Theorem 17. If $\Omega$ is a prefect positive correlation probability space and $\widetilde{H}$ is a falling fuzzy left (right) $h$-ideal of $S$ for all $x, y, a, b, z \in S$, then

(1) $\widetilde{H}(x+y) \geq \min \{\widetilde{H}(x), \widetilde{H}(y)\}$;

(2) $\widetilde{H}(x y) \geq \widetilde{H}(y)(\widetilde{H}(x y) \geq \widetilde{H}(x))$;

(3) $x+a+z=b+z \Rightarrow \widetilde{H}(x) \geq \min \{\widetilde{H}(a), \widetilde{H}(b)\}$.

Proof. (1) By Definition 4, $\xi(\omega)$ is a left $h$-ideal of $S$ for any $\omega \in \Omega$. Hence by Theorem $10, \Omega(x ; \xi) \cap \Omega(y ; \xi) \subseteq \Omega(x+y ; \xi)$.

Thus, we have $\widetilde{H}(x+y)=P(\omega \mid x+y \in \xi(\omega)) \geq P(\{\omega \mid$ $x \in \xi(\omega)\} \cap\{\omega \mid y \in \xi(\omega)\})$.

If $\{\omega \mid x \in \xi(\omega)\} \supseteq\{\omega \mid y \in \xi(\omega)\}$, then $\widetilde{H}(x+y) \geq \widetilde{H}(x)$. If $\{\omega \mid x \in \xi(\omega)\} \subseteq\{\omega \mid y \in \xi(\omega)\}$, then $\widetilde{H}(x+y) \geq \widetilde{H}(y)$, and so $\widetilde{H}(x+y) \geq \min \{\widetilde{H}(x), \widetilde{H}(y)\}$.

(2) By Definition 4, $\xi(\omega)$ is a left $h$-ideal of $S$ for any $\omega \in \Omega$. Hence by Theorem $10, \Omega(y ; \xi) \subseteq \Omega(x y$; $\xi)$. Thus, we have

$$
\begin{aligned}
\widetilde{H}(x y) & =P(\omega \mid x y \in \xi(\omega)) \\
& \geq P(\omega \mid y \in \xi(\omega)) \\
& =\widetilde{H}(y) .
\end{aligned}
$$

(3) By Definition $4, \xi(\omega)$ is a left $h$-ideal of $S$ for any $\omega \in \Omega$. Hence by Theorem $10, x+a+z=b+z \Rightarrow \Omega(a ; \xi) \cap \Omega(b ; \xi) \subseteq$ $\Omega(x ; \xi)$. Thus, we have $\widetilde{H}(x)=P(\omega \mid x \in \xi(\omega)) \geq P(\{\omega \mid a \in$ $\xi(\omega)\} \cap\{\omega \mid b \in \xi(\omega)\})$.

If $\{\omega \mid a \in \xi(\omega)\} \supseteq\{\omega \mid b \in \xi(\omega)\}$, then $\widetilde{H}(x) \geq \widetilde{H}(a)$. If $\{\omega \mid a \in \xi(\omega)\} \subseteq\{\omega \mid b \in \xi(\omega)\}$, then $\widetilde{H}(x) \geq \widetilde{H}(b)$, and so $\widetilde{H}(x) \geq \min \{\widetilde{H}(a), \widetilde{H}(b)\}$.

Proposition 18 (see [5]). If $\Omega$ is a prefect positive correlation probability space, then $\left(\widetilde{H}_{1} \cap \widetilde{H}_{2}\right)(x)=\min \left\{\widetilde{H}_{1}(x), \widetilde{H}_{2}(x)\right\}$. 
Proposition 19. If $\Omega$ is a prefect positive correlation probability space and $\widetilde{H}_{1}$ and $\widetilde{H}_{2}$ are two falling fuzzy left (right) $h$ ideals of $S$, then $\widetilde{H}_{1} \cap \widetilde{H}_{2}$ is a falling fuzzy left (right) $h$-ideal of $S$.

Proof. We only consider the case of left $h$-ideals, and the proof of right $h$-ideals is similar:

(i)

$$
\begin{aligned}
\left(\widetilde{H}_{1} \cap \widetilde{H}_{2}\right)(x+y)= & \min \left\{\widetilde{H}_{1}(x+y), \widetilde{H}_{2}(x+y)\right\} \\
\geq & \min \left\{\min \left\{\widetilde{H}_{1}(x), \widetilde{H}_{1}(y)\right\},\right. \\
& \left.\min \left\{\widetilde{H}_{2}(x), \widetilde{H}_{2}(y)\right\}\right\} \\
= & \min \left\{\min \left\{\widetilde{H}_{1}(x), \widetilde{H}_{2}(x)\right\},\right. \\
& \left.\min \left\{\widetilde{H}_{1}(y), \widetilde{H}_{2}(y)\right\}\right\} \\
= & \min \left\{\left(\widetilde{H}_{1} \cap \widetilde{H}_{2}\right)(x),\right. \\
& \left.\left(\widetilde{H}_{1} \cap \widetilde{H}_{2}\right)(y)\right\},
\end{aligned}
$$

(ii)

$$
\begin{aligned}
\left(\widetilde{H}_{1} \cap \widetilde{H}_{2}\right)(x y) & =\min \left\{\widetilde{H}_{1}(x y), \widetilde{H}_{2}(x y)\right\} \\
& \geq \min \left\{\widetilde{H}_{1}(y), \widetilde{H}_{2}(y)\right\} \\
& =\left(\widetilde{H}_{1} \cap \widetilde{H}_{2}\right)(y),
\end{aligned}
$$

(iii)

$$
\begin{aligned}
x+a+z=b+z \Longrightarrow\left(\widetilde{H}_{1} \cap \widetilde{H}_{2}\right)(x) \\
\geq \min \left\{\min \left\{\widetilde{H}_{1}(a), \widetilde{H}_{1}(b)\right\},\right. \\
\left.\min \left\{\widetilde{H}_{2}(a), \widetilde{H}_{2}(b)\right\}\right\} \\
=\min \left\{\min \left\{\widetilde{H}_{1}(a), \widetilde{H}_{2}(a)\right\},\right. \\
\left.\min \left\{\widetilde{H}_{1}(b), \widetilde{H}_{2}(b)\right\}\right\} \\
=\min \left\{\left(\widetilde{H}_{1} \cap \widetilde{H}_{2}\right)(a),\left(\widetilde{H}_{1} \cap \widetilde{H}_{2}\right)(b)\right\} .
\end{aligned}
$$

Theorem 20. If a falling fuzzy set $\widetilde{H}$ of $S$ is a falling fuzzy left (right) $h$-ideal of $S$, then $\chi_{S} \odot_{p} \widetilde{H} \subseteq \widetilde{H}\left(\widetilde{H} \odot_{p} \chi_{S} \subseteq \widetilde{H}\right)$.

Proof. We only consider the case of left $h$-ideals, and the proof of right $h$-ideals is similar. It is sufficient to show that the condition is satisfied. Let $x \in S$. If $\left(\chi_{S} \odot_{p} \widetilde{H}\right)(x)=0$, it is clear that $\left(\chi_{S} \odot_{p} \widetilde{H}\right)(x) \leq \widetilde{H}(x)$. Otherwise, there exist $a_{i}, b_{i}, a_{j}^{\prime}, b_{j}^{\prime} \epsilon$ $S$ such that $x+\sum_{i=1}^{m} a_{i} b_{i}+z=\sum_{j=1}^{n} a_{j}^{\prime} b_{j}^{\prime}+z$. Then we have

$$
\begin{aligned}
& \left(\chi_{S} \odot_{p} \widetilde{H}\right)(x) \\
& \quad=\sup _{x+\sum_{i=1}^{m} a_{i} b_{i}+z=\sum_{j=1}^{n} a_{j}^{\prime} b_{j}^{\prime}+z}\left(\min \left\{\widetilde{H}\left(b_{i}\right), \widetilde{H}\left(b_{j}^{\prime}\right)\right\}\right) \\
& \quad \leq \sup _{x+\sum_{i=1}^{m} a_{i} b_{i}+z=\sum_{j=1}^{n} a_{j}^{\prime} b_{j}^{\prime}+z}\left(\min \left\{\widetilde{H}\left(a_{i} b_{i}\right), \widetilde{H}\left(a_{j}^{\prime} b_{j}^{\prime}\right)\right\}\right) \\
& \quad \leq \sup _{x+\sum_{i=1}^{m} a_{i} b_{i}+z=\sum_{j=1}^{n} a_{j}^{\prime} b_{j}^{\prime}+z}\left(\operatorname { m i n } \left\{\widetilde{H}\left(\sum_{i=1}^{m} a_{i} b_{i}\right),\right.\right. \\
& \left.\left.\quad \widetilde{H}\left(\sum_{j=1}^{n} a_{j}^{\prime} b_{j}^{\prime}\right)\right\}\right) \\
& \quad=\quad \sup _{x+\sum_{i=1}^{m} a_{i} b_{i}+z=\sum_{j=1}^{n} a_{j}^{\prime} b_{j}^{\prime}+z} \widetilde{H}(x) \\
& =\widetilde{H}(x) .
\end{aligned}
$$

This implies that $\chi_{S} \odot_{p} \widetilde{H} \subseteq \widetilde{H}$.

Theorem 21. Let $S$ be a hemiring and $A, B \subseteq S$. Then $\chi_{A} \odot_{p} \chi_{B}=\chi_{\overline{A B}}$.

Proof. Let $x \in S$. If $x \in \overline{A B}$, then $\chi_{\overline{A B}}(x)=1$ and $x+$ $\sum_{i=1}^{m} p_{i} q_{i}+z=\sum_{j=1}^{n} p_{j}^{\prime} q_{j}^{\prime}+z$ for some $p_{i}, p_{j}^{\prime} \in A, q_{i}, q_{j}^{\prime} \in B$ and $z \in S$. Thus we have

$$
\begin{aligned}
& \left(\chi_{A} \odot_{p} \chi_{B}\right)(x) \\
& =\sup _{x+\sum_{i=1}^{m} a_{i} b_{i}+z=\sum_{j=1}^{n} a_{j}^{\prime} b_{j}^{\prime}+z}\left(\operatorname { m i n } \left\{\chi_{A}\left(a_{i}\right), \chi_{A}\left(a_{j}^{\prime}\right),\right.\right. \\
& \left.\left.\chi_{B}\left(b_{i}\right), \chi_{B}\left(b_{j}^{\prime}\right)\right\}\right) \\
& \geq \min \left\{\chi_{A}\left(p_{i}\right), \chi_{A}\left(p_{j}^{\prime}\right), \chi_{B}\left(q_{i}\right), \chi_{B}\left(q_{j}^{\prime}\right)\right\} \\
& =1,
\end{aligned}
$$

and so $\left(\chi_{A} \odot_{p} \chi_{B}\right)(x)=1=\chi_{\overline{A B}}(x)$. Then

If $x \notin \overline{A B}$, then $\chi_{\overline{A B}}(x)$. If possible, let $\left(\chi_{A} \odot_{p} \chi_{B}\right)(x) \neq 0$.

$$
\begin{array}{r}
\left(\chi_{A} \odot_{p} \chi_{B}\right)(x) \\
=\sup _{x+\sum_{i=1}^{m} a_{i} b_{i}+z=\sum_{j=1}^{n} a_{j}^{\prime} b_{j}^{\prime}+z}\left(\operatorname { m i n } \left\{\chi_{A}\left(a_{i}\right), \chi_{A}\left(a_{j}^{\prime}\right),\right.\right. \\
\left.\left.\chi_{B}\left(b_{i}\right), \chi_{B}\left(b_{j}^{\prime}\right)\right\}\right)
\end{array}
$$

$\neq 0$. 
Hence, there exist $p_{i}, p_{j}^{\prime}, q_{i}, q_{j}^{\prime}, z \in S$ such that $x+$ $\sum_{i=1}^{m} p_{i} q_{i}+z=\sum_{j=1}^{n} p_{j}^{\prime} q_{j}^{\prime}+z$ and $\min \left\{\chi_{A}\left(p_{i}\right), \chi_{A}\left(p_{j}^{\prime}\right), \chi_{B}\left(q_{i}\right)\right.$, $\left.\chi_{B}\left(q_{j}^{\prime}\right)\right\} \neq 0$, that is, $\chi_{A}\left(p_{i}\right)=\chi_{A}\left(p_{j}^{\prime}\right)=\chi_{B}\left(q_{i}\right)=\chi_{B}\left(q_{j}^{\prime}\right)=1$, hence $p_{i}, p_{j}^{\prime} \in A, q_{i}, q_{j}^{\prime} \in B$ and $x \in \overline{A B}$, which contradicts with $\chi_{\overline{A B}}(x)=0$. Thus we have $\left(\chi_{A} \odot_{p} \chi_{B}\right)(x)=0=\chi_{\overline{A B}}(x)$.

In any case, we have $\left(\chi_{A} \odot_{p} \chi_{B}\right)(x)=\chi_{\overline{A B}}(x)$. This completes the proof.

Theorem 22. A hemiring $S$ is h-hemiregular if and only if for any falling fuzzy right $h$-ideal $\widetilde{H}_{1}$ and any falling fuzzy left $h$ ideal $\widetilde{H}_{2}$ of $S$ we have $\widetilde{H}_{1} \odot_{p} \widetilde{H}_{2}=\widetilde{H}_{1} \cap \widetilde{H}_{2}$.

Proof. Let $S$ be an $h$-hemiregular hemiring, $\widetilde{H}_{1}$ and $\widetilde{H}_{2}$ be any falling fuzzy right $h$-ideal and any falling fuzzy left $h$-ideal of $S$, respectively. Then by Theorem 21, we have $\widetilde{H}_{1} \odot_{p} \widetilde{H}_{2} \subseteq$ $\widetilde{H}_{1} \odot_{p} \chi_{S} \subseteq \widetilde{H}_{1}$ and $\widetilde{H}_{1} \odot_{p} \widetilde{H}_{2} \subseteq \chi_{S} \odot_{p} \widetilde{H}_{2} \subseteq \widetilde{H}_{2}$. Thus $\widetilde{H}_{1} \odot_{p} \widetilde{H}_{2} \subseteq$ $\widetilde{H}_{1} \cap \widetilde{H}_{2}$. To show the converse inclusion, let $x$ be any element of $S$. Since $S$ is $h$-hemiregular, there exist $a, a^{\prime}, z \in S$ such that $x+x a x+z=x a^{\prime} x+z$. Then we have

$$
\begin{aligned}
& \left(\widetilde{H}_{1} \odot_{p} \widetilde{H}_{2}\right)(x) \\
& =\sup _{x+\sum_{i=1}^{m} a_{i} b_{i}+z=\sum_{j=1}^{n} a_{j}^{\prime} b_{j}^{\prime}+z}\left(\operatorname { m i n } \left\{\widetilde{H}_{1}\left(a_{i}\right), \widetilde{H}_{1}\left(a_{j}^{\prime}\right), \widetilde{H}_{2}\left(b_{i}\right),\right.\right. \\
& \qquad \widetilde{H}_{2}\left(b_{j}^{\prime}\right) \mid i=1, \ldots, m, \\
& \quad j=1, \ldots, n\}) \\
& \geq \min \left\{\widetilde{H}_{1}(x a), \widetilde{H}_{1}\left(x a^{\prime}\right), \widetilde{H}_{2}(x)\right\} \\
& \geq \min \left\{\widetilde{H}_{1}(x), \widetilde{H}_{2}(x)\right\} \\
& =\left(\widetilde{H}_{1} \cap \widetilde{H}_{2}\right)(x) .
\end{aligned}
$$

This implies that $\widetilde{H}_{1} \odot_{p} \widetilde{H}_{2} \supseteq \widetilde{H}_{1} \cap \widetilde{H}_{2}$. Therefore, $\widetilde{H}_{1} \odot_{p} \widetilde{H}_{2}=\widetilde{H}_{1} \cap \widetilde{H}_{2}$.

Conversely, let $\xi_{1}(\omega)$ and $\xi_{2}(\omega)$ be any right $h$-ideal and any left $h$-ideal of $S$, respectively. Then by Definition $4, \widetilde{H}_{1}(\mu)$ and $\widetilde{H}_{2}(\mu)$ are any falling right $h$-ideal and any falling left fuzzy $h$-ideal of $S$, respectively. The characteristic functions $\chi_{\xi_{1}(\omega)}$ and $\chi_{\xi_{2}(\omega)}$ are a fuzzy right $h$-ideal and a fuzzy left $h$ ideal of $S$, respectively. Now, by Theorem 21 , we have

$$
\chi_{\overline{\xi_{1}(\omega) \xi_{2}(\omega)}}=\chi_{\xi_{1}(\omega)} \odot_{p} \chi_{\xi_{2}(\omega)}=\chi_{\xi_{1}(\omega)} \cap \chi_{\xi_{2}(\omega)}=\chi_{\xi_{1}(\omega) \cap \xi_{2}(\omega)} .
$$

It follows from Theorem 21 that $\overline{\xi_{1}(\omega) \xi_{2}(\omega)}=\xi_{1}(\omega) \cap$ $\xi_{2}(\omega)$. Thus, we have

$$
\begin{aligned}
\overline{\left(\widetilde{H}_{1} \widetilde{H}_{2}\right)}(\mu) & =P\left(\omega \mid \mu \in \overline{\xi_{1}(\omega) \xi_{2}(\omega)}\right) \\
& =P\left(\omega \mid \mu \in \xi_{1}(\omega) \cap \xi_{2}(\omega)\right) \\
& =\left(\widetilde{H}_{1} \cap \widetilde{H}_{2}\right)(\mu) .
\end{aligned}
$$

Therefore $S$ is $h$-hemiregular by Lemma 14 .
4.2. Independent Probability Spaces. In this subsection, we describe the characterizations of $h$-hemiregular hemirings based on independent probability spaces via falling fuzzy $h$ ideals.

Definition 23. The probability space $\Omega$ is called independent if $\Omega(x ; \xi) \cap \Omega(y ; \xi)=\Omega(x ; \xi) \Omega(y ; \xi)$ for all $x, y \in S$.

Definition 24 . If $\Omega$ is an independent probability space and let $\widetilde{H}_{1}$ and $\widetilde{H}_{2}$ be falling fuzzy $h$-ideals of $S$. Then the $I$-product of $\widetilde{H}_{1}$ and $\widetilde{H}_{2}$ is defined by

$$
\begin{aligned}
& \left(\widetilde{H}_{1} \odot_{i} \widetilde{H}_{2}\right)(x) \\
& =\left\{\begin{array}{c}
\widetilde{H}_{1}\left(a_{i}\right) \widetilde{H}_{1}\left(a_{j}^{\prime}\right) \widetilde{H}_{2}\left(b_{i}\right) \widetilde{H}_{2}\left(b_{j}^{\prime}\right), \\
\quad \text { if } x+\sum_{i=1}^{m} a_{i} b_{i}+z=\sum_{j=1}^{n} a_{j}^{\prime} b_{j}^{\prime}+z ; \\
0, \quad \text { otherwise. }
\end{array}\right.
\end{aligned}
$$

Theorem 25. If $\Omega$ is an independent probability space and $\widetilde{H}$ is a falling fuzzy left (right) $h$-ideal of $S$ for all $x, y, a, b, z \in S$, then
(1) $\widetilde{H}(x+y) \geq \widetilde{H}(x) \widetilde{H}(y)$;
(2) $\widetilde{H}(x y) \geq \widetilde{H}(y)(\widetilde{H}(x y) \geq \widetilde{H}(x))$;
(3) $x+a+z=b+z \Rightarrow \widetilde{H}(x) \geq \widetilde{H}(a) \widetilde{H}(b)$.

Proof. (1) By Definition 4, $\xi(\omega)$ is a left $h$-ideal of $S$ for any $\omega \in \Omega$. Hence by Theorem $10, \Omega(x ; \xi) \cap \Omega(y ; \xi) \subseteq \Omega(x+y ; \xi)$.

Thus, we have

$$
\begin{aligned}
\widetilde{H}(x+y) & =P(\omega \mid x+y \in \xi(\omega)) \\
& \geq P(\{\omega \mid x \in \xi(\omega)\} \cap\{\omega \mid y \in \xi(\omega)\}) \\
& =P(\omega \mid x \in \xi(\omega)) P(\omega \mid y \in \xi(\omega)) \\
& =\widetilde{H}(x) \widetilde{H}(y) .
\end{aligned}
$$

(2) By Definition $4, \xi(\omega)$ is a left $h$-ideal of $S$ for any $\omega \in \Omega$. Hence by Theorem $10, \Omega(y ; \xi) \subseteq \Omega(x y$; $\xi$. Thus, we have

$$
\begin{aligned}
\widetilde{H}(x y) & =P(\omega \mid x y \in \xi(\omega)) \\
& \geq P(\omega \mid y \in \xi(\omega)) \\
& =\widetilde{H}(y) .
\end{aligned}
$$

(3) By Definition $4, \xi(\omega)$ is a left $h$-ideal of $S$ for any $\omega \in \Omega$. Hence by Theorem $10, x+a+z=b+z \Rightarrow \Omega(a ; \xi) \cap \Omega(b ; \xi) \subseteq$ $\Omega(x ; \xi)$. Thus, we have

$$
\begin{aligned}
\widetilde{H}(x) & =P(\omega \mid x \in \xi(\omega)) \\
& \geq P(\{\omega \mid a \in \xi(\omega)\} \cap\{\omega \mid b \in \xi(\omega)\}) \\
& =P(\omega \mid a \in \xi(\omega)) P(\omega \mid b \in \xi(\omega)) \\
& =\widetilde{H}(a) \widetilde{H}(b) .
\end{aligned}
$$


Proposition 26 (see [5]). If $\Omega$ is an independent probability space, then $\left(\widetilde{H}_{1} \cap \widetilde{H}_{2}\right)(x)=\widetilde{H}_{1}(x) \widetilde{H}_{2}(x)$.

Proposition 27. If $\Omega$ is an independent probability space and $\widetilde{H}$ is a falling fuzzy $h$-ideal of S, then $\widetilde{H}(x y) \geq \widetilde{H}(x)+\widetilde{H}(y)-$ $\widetilde{H}(x) \widetilde{H}(y)$.

Proof. Since $\Omega(x y ; \xi) \supseteq \Omega(x ; \xi) \cap \Omega(y ; \xi)$, it follows that

$$
\begin{aligned}
\widetilde{H}(x y)= & P(\omega \mid x y \in \xi(\omega)) \\
\geq & P((\omega \mid x \in \xi(\omega)) \cap(\omega \mid y \in \xi(\omega))) \\
\geq & P(\omega \mid x \in \xi(\omega))+P(\omega \mid y \in \xi(\omega)) \\
& -P((\omega \mid x \in \xi(\omega)) \cap(\omega \mid y \in \xi(\omega))) \\
= & P(\omega \mid x \in \xi(\omega))+P(\omega \mid y \in \xi(\omega)) \\
& -P(\omega \mid x \in \xi(\omega)) P(\omega \mid y \in \xi(\omega)) \\
= & \widetilde{H}(x)+\widetilde{H}(y)-\widetilde{H}(x) \widetilde{H}(y) .
\end{aligned}
$$

Proposition 28. Let $\Omega$ be an independent probability space and $\widetilde{H}_{1}$ and $\widetilde{H}_{2}$ be two falling fuzzy left (right) $h$-ideals of $S$, and then $\widetilde{H}_{1} \cap \widetilde{H}_{2}$ is a falling fuzzy left (right) $h$-ideal of S.

Proof. We only consider the case of left $h$-ideals, and the proof of right $h$-ideals is similar:

(i)

$$
\begin{aligned}
\left(\widetilde{H}_{1} \cap \widetilde{H}_{2}\right)(x+y) & =\widetilde{H}_{1}(x+y) \widetilde{H}_{2}(x+y) \\
& \geq \widetilde{H}_{1}(x) \widetilde{H}_{1}(y) \widetilde{H}_{2}(x) \widetilde{H}_{2}(y) \\
& =\left(\widetilde{H}_{1} \cap \widetilde{H}_{2}\right)(x)\left(\widetilde{H}_{1} \cap \widetilde{H}_{2}\right)(y),
\end{aligned}
$$

(ii)

$$
\begin{aligned}
\left(\widetilde{H}_{1} \cap \widetilde{H}_{2}\right)(x y) & =\widetilde{H}_{1}(x y) \widetilde{H}_{2}(x y) \\
& \geq \widetilde{H}_{1}(y) \widetilde{H}_{2}(y) \\
& =\left(\widetilde{H}_{1} \cap \widetilde{H}_{2}\right)(y),
\end{aligned}
$$

(iii)

$$
\begin{aligned}
x+a+z & =b+z \Longrightarrow\left(\widetilde{H}_{1} \cap \widetilde{H}_{2}\right)(x) \\
& \geq \widetilde{H}_{1}(a) \widetilde{H}_{1}(b) \widetilde{H}_{2}(a) \widetilde{H}_{2}(b) \\
& =\left(\widetilde{H}_{1} \cap \widetilde{H}_{2}\right)(a)\left(\widetilde{H}_{1} \cap \widetilde{H}_{2}\right)(b) .
\end{aligned}
$$

Theorem 29. If a falling fuzzy set $\widetilde{H}$ of $S$ is a falling fuzzy left (right) $h$-ideal of $S$, then $\chi_{S} \odot_{i} \widetilde{H} \subseteq \widetilde{H}\left(\widetilde{H} \odot_{i} \chi_{S} \subseteq \widetilde{H}\right)$.
Proof. We only consider the case of left $h$-ideals, and the proof of right $h$-ideals is similar. It is sufficient to show that the condition is satisfied. Let $x \in S$. If $\left(\chi_{S} \odot_{i} \widetilde{H}\right)(x)=0$, it is clear that $\left(\chi_{S} \odot_{i} \widetilde{H}\right)(x) \leq \widetilde{H}(x)$. Otherwise, there exist $a_{i}, b_{i}, a_{j}^{\prime}, b_{j}^{\prime} \epsilon$ $S$ such that $x+\sum_{i=1}^{m} a_{i} b_{i}+z=\sum_{j=1}^{n} a_{j}^{\prime} b_{j}^{\prime}+z$. Then we have

$$
\begin{aligned}
\left(\chi_{S} \odot_{i} \widetilde{H}\right)(x) & =\widetilde{H}\left(b_{i}\right) \widetilde{H}\left(b_{j}^{\prime}\right) \\
& \leq \widetilde{H}\left(a_{i} b_{i}\right) \widetilde{H}\left(a_{j}^{\prime} b_{j}^{\prime}\right) \\
& \leq \widetilde{H}\left(\sum_{i=1}^{m} a_{i} b_{i}\right) \widetilde{H}\left(\sum_{j=1}^{n} a_{j}^{\prime} b_{j}^{\prime}\right) \\
& \leq \widetilde{H}(x) .
\end{aligned}
$$

This implies that $\chi_{S} \odot_{i} \widetilde{H} \subseteq \widetilde{H}$.

Theorem 30. Let $S$ be a hemiring and $A, B \subseteq S$, and then $\chi_{A} \odot_{i} \chi_{B}=\chi_{\overline{A B}}$.

Proof. Let $x \in S$. If $x \in \overline{A B}$, then $\chi_{\overline{A B}}(x)=1$ and $x+$ $\sum_{i=1}^{m} p_{i} q_{i}+z=\sum_{j=1}^{n} p_{j}^{\prime} q_{j}^{\prime}+z$ for some $p_{i}, p_{j}^{\prime} \in A, q_{i}, q_{j}^{\prime} \in B$ and $z \in S$. Thus we have

$$
\begin{aligned}
\left(\chi_{A} \odot_{i} \chi_{B}\right)(x) & =\chi_{A}\left(a_{i}\right) \chi_{A}\left(a_{j}^{\prime}\right) \chi_{B}\left(b_{i}\right) \chi_{B}\left(b_{j}^{\prime}\right) \\
& \geq \chi_{A}\left(p_{i}\right) \chi_{A}\left(p_{j}^{\prime}\right) \chi_{B}\left(q_{i}\right) \chi_{B}\left(q_{j}^{\prime}\right) \\
& =1,
\end{aligned}
$$

and so $\left(\chi_{A} \odot_{i} \chi_{B}\right)(x)=1=\chi_{\overline{A B}}(x)$. 0 . Then

If $x \notin \overline{A B}$, then $\chi_{\overline{A B}}(x)=0$. If possible, let $\left(\chi_{A} \odot_{i} \chi_{B}\right)(x) \neq$

$$
\left(\chi_{A} \odot_{i} \chi_{B}\right)(x)=\chi_{A}\left(a_{i}\right) \chi_{A}\left(a_{j}^{\prime}\right) \chi_{B}\left(b_{i}\right) \chi_{B}\left(b_{j}^{\prime}\right) \neq 0 .
$$

Hence, there exist $p_{i}, p_{j}^{\prime}, q_{i}, q_{j}^{\prime}, z \in S$ such that $x+$ $\sum_{i=1}^{m} p_{i} q_{i}+z=\sum_{j=1}^{n} p_{j}^{\prime} q_{j}^{\prime}+z$ and $\chi_{A}\left(p_{i}\right) \chi_{A}\left(p_{j}^{\prime}\right) \chi_{B}\left(q_{i}\right) \chi_{B}\left(q_{j}^{\prime}\right) \neq$ 0 , that is, $\chi_{A}\left(p_{i}\right)=\chi_{A}\left(p_{j}^{\prime}\right)=\chi_{B}\left(q_{i}\right)=\chi_{B}\left(q_{j}^{\prime}\right)=1$; hence, $p_{i}, p_{j}^{\prime} \in A, q_{i}, q_{j}^{\prime} \in B$ and $x \in \overline{A B}$, which contradicts with $\chi_{\overline{A B}}(x)=0$. Thus we have $\left(\chi_{A} \odot_{i} \chi_{B}\right)(x)=0=\chi_{\overline{A B}}(x)$.

In any case, we have $\left(\chi_{A} \odot_{i} \chi_{B}\right)(x)=\chi_{\overline{A B}}(x)$. This completes the proof.

Theorem 31. A hemiring $S$ is h-hemiregular if and only if for any falling fuzzy right $h$-ideal $\widetilde{H}_{1}$ and any falling fuzzy left $h$ ideal $\widetilde{H}_{2}$ of $S, \widetilde{H}_{1} \odot_{i} \widetilde{H}_{2}=\widetilde{H}_{1} \cap \widetilde{H}_{2}$.

Proof. (1) Let $S$ be an $h$-hemiregular hemiring, $\widetilde{H}_{1}$ any falling fuzzy right $h$-ideal, and $\widetilde{H}_{2}$ any falling fuzzy left $h$-ideal of $S$, respectively. Then by Theorem 29 , we have $\widetilde{H}_{1} \odot_{i} \widetilde{H}_{2} \subseteq$ $\widetilde{H}_{1} \odot_{i} \chi_{S} \subseteq \widetilde{H}_{1}$ and $\widetilde{H}_{1} \odot_{i} \widetilde{H}_{2} \subseteq \chi_{S} \odot_{i} \widetilde{H}_{2} \subseteq \widetilde{H}_{2}$. Thus $\widetilde{H}_{1} \odot_{i} \widetilde{H}_{2} \subseteq$ $\widetilde{H}_{1} \cap \widetilde{H}_{2}$. To show the converse inclusion, let $x$ be any element 
of $S$. Since $S$ is $h$-hemiregular, there exist $a, a^{\prime}, z \in S$ such that $x+x a x+z=x a^{\prime} x+z$. Then we have

$$
\begin{aligned}
\widetilde{H}_{1} \odot_{i} \widetilde{H}_{2}(x) & =\widetilde{H}_{1}\left(a_{i}\right) \widetilde{H}_{1}\left(a_{j}^{\prime}\right) \widetilde{H}_{2}\left(b_{i}\right) \widetilde{H}_{2}\left(b_{j}^{\prime}\right) \\
& \geq \widetilde{H}_{1}(x a x) \widetilde{H}_{1}\left(x a^{\prime} x\right) \\
& \geq \widetilde{H}_{1}(a x) \widetilde{H}_{2}\left(a^{\prime} x\right) \\
& \geq \widetilde{H}_{1}(x) \widetilde{H}_{2}(x) \\
& =\left(\widetilde{H}_{1} \cap \widetilde{H}_{2}\right)(x) .
\end{aligned}
$$

This implies that $\widetilde{H}_{1} \widetilde{\odot}_{i} \widetilde{H}_{2} \supseteq \widetilde{H}_{1} \cap \widetilde{H}_{2}$. Therefore, we have $\widetilde{H}_{1} \widetilde{\odot}_{i} \widetilde{H}_{2}=\widetilde{H}_{1} \cap \widetilde{H}_{2}$.

Conversely, let $\xi_{1}(\omega)$ and $\xi_{2}(\omega)$ be any right $h$-ideal and any left $h$-ideal of $S$, respectively. Then by Definition $4, \widetilde{H}_{1}(\mu)$ and $\widetilde{H}_{2}(\mu)$ are a falling right $h$-ideal and a falling left fuzzy $h$ ideal of $S$, respectively. The characteristic functions $\chi_{\xi_{1}(\omega)}$ and $\chi_{\xi_{2}(\omega)}$ of $\xi_{1}(\omega)$ and $\xi_{2}(\omega)$ are a fuzzy right $h$-ideal and a fuzzy left $h$-ideal of $S$, respectively. Now, by Theorem 30, we have

$$
\chi_{\overline{\xi_{1}(\omega) \xi_{2}(\omega)}}=\chi_{\xi_{1}(\omega)} \odot_{i} \chi_{\xi_{2}(\omega)}=\chi_{\xi_{1}(\omega)} \cap \chi_{\xi_{2}(\omega)}=\chi_{\xi_{1}(\omega) \cap \xi_{2}(\omega)} .
$$

It follows from Theorem 30 that $\overline{\xi_{1}(\omega) \xi_{2}(\omega)}=\xi_{1}(\omega) \cap$ $\xi_{2}(\omega)$. So $\overline{\left(\widetilde{H}_{1} \widetilde{H}_{2}\right)}(\mu)=P\left(\omega \mid \mu \in \overline{\xi_{1}(\omega) \xi_{2}(\omega)}\right)=P(\omega \mid \mu \epsilon$ $\left.\xi_{1}(\omega) \cap \xi_{2}(\omega)\right)=\left(\widetilde{H}_{1} \cap \widetilde{H}_{2}\right)(\mu)$.

Therefore, $S$ is $h$-hemiregular by Lemma 14 .

\section{Conclusions}

In this paper, we introduce the notion of falling fuzzy $h$-ideals of a hemiring. Then we investigate some characteristics of $h$-hemiregular by means of falling fuzzy $h$-ideals based on independent (prefect positive correlation) probability spaces. In future work, one can consider $h$-hemiregular using falling fuzzy $h$-bi-ideals and falling fuzzy $h$-quasi-ideals. One also can apply fuzzy inference relations to hemirings. Further, one can investigate this theory to information sciences and intelligent and fuzzy systems.

\section{Acknowledgments}

The authors are extremely grateful to the referees and Editor: Professor Qiankun Song for giving them many valuable comments and helpful suggestions which helped them improve the presentation of this paper. This research is partially supported by a Grant of National Natural Science Foundation of China, (61175055), Innovation Term of Higher Education of Hubei Province, China, (T201109), Natural Science Foundation of Hubei Province (2012FFB01101), and Natural Science Foundation of Education Committee of Hubei Province (D20131903).

\section{References}

[1] L. A. Zadeh, "Fuzzy sets," Information and Computation, vol. 8, pp. 338-353, 1965.
[2] I. R. Goodman, "Fuzzy sets as equivalence classes of random sets," in Recent Developments in Fuzzy Sets and Possibility Theory, R. Yager, Ed., Pergamon, New York, NY, USA, 1982.

[3] P. Z. Wang and E. Sanchez, "Treating a fuzzy subset as a projectable random set," in Fuzzy Information and Decision, M. M. Gupta and E. Sanchez, Eds., pp. 212-219, Pergamon, New York, NY, USA, 1982.

[4] S. K. Tan, P. Z. Wang, and E. S. Lee, "Fuzzy set operations based on the theory of falling shadows," Journal of Mathematical Analysis and Applications, vol. 174, no. 1, pp. 242-255, 1993.

[5] S. K. Tan, P. Z. Wang, and X. Z. Zhang, "Fuzzy inference relation based on the theory of falling shadows," Fuzzy Sets and Systems, vol. 53, no. 2, pp. 179-188, 1993.

[6] X.-H. Yuan and E. S. Lee, "A fuzzy algebraic system based on the theory of falling shadows," Journal of Mathematical Analysis and Applications, vol. 208, no. 1, pp. 243-251, 1997.

[7] Y. B. Jun and M. S. Kang, "Fuzzy positive implicative ideals of BCK-algebras based on the theory of falling shadows," Computers and Mathematics with Applications, vol. 61, no. 1, pp. 62-67, 2011.

[8] L. Beasley and N. J. Pullman, "Linear operators strongly preserving idempotent matrices over semirings," Linear Algebra and Its Applications, vol. 160, pp. 217-229, 1992.

[9] S. Ghosh, "Matrices over semirings," Information Sciences, vol. 90, no. 1-4, pp. 221-230, 1996.

[10] D. R. LaTorre, "On $h$-ideals and $k$-ideals in hemirings," Publicationes Mathematicae Debrecen, vol. 12, pp. 219-226, 1965.

[11] S. Abdullah, B. Davvaz, and M. Aslam, " $(\alpha, \beta)$-intuitionistic fuzzy ideals of hemirings," Computers and Mathematics with Applications, vol. 62, no. 8, pp. 3077-3090, 2011.

[12] J. Ahsan, K. Saifullah, and M. F. Khan, "Fuzzy semirings," Fuzzy Sets and Systems, vol. 60, no. 3, pp. 309-320, 1993.

[13] J. Ahsan, J. N. Mordeson, and M. Shabir, "Fuzzy $k$-ideals of semirngs," in Fuzzy Semiring With Applications Automata Theory, vol. 278 of Studies in Fuzziness and Soft Computing, pp. 5382, 2012.

[14] T. K. Dutta and B. K. Biswas, "Fuzzy congruence and quotient semiring of a semiring," Journal of Fuzzy Mathematics, vol. 4, no. 4, pp. 737-748, 1996.

[15] H. Hedayati, "Fuzzy ideals of semirings," in Neural Computing and Applications, vol. 20, pp. 1219-1228, 2011.

[16] Y. B. Jun, H. S. Kim, and M. A. Öztürk, "Fuzzy $k$-ideals in semirings," Journal of Fuzzy Mathematics, vol. 13, no. 2, pp. 351364, 2005.

[17] J. Zhan and W. A. Dudek, "Fuzzy $h$-ideals of hemirings," Information Sciences, vol. 177, no. 3, pp. 876-886, 2007.

[18] Y. Q. Yin and H. X. Li, “The characterizations of $h$-hemiregular hemirings and $h$-intra-hemiregular hemirings," Information Sciences, vol. 178, no. 17, pp. 3451-3464, 2008.

[19] Y. Q. Yin, X. K. Huang, D. H. Xu, and H. J. Li, “The characterization of $h$-semisimple hemirings," International Journal of Fuzzy Systems, vol. 11, no. 2, pp. 116-122, 2009.

[20] W. A. Dudek, M. Shabir, and M. Irfan Ali, “ $(\alpha, \beta)$-fuzzy ideals of hemirings," Computers and Mathematics with Applications, vol. 58, no. 2, pp. 310-321, 2009.

[21] W. A. Dudek, M. Shabir, and R. Anjum, "Characterizations of hemirings by their $h$-ideals," Computers and Mathematics with Applications, vol. 59, no. 9, pp. 3167-3179, 2010.

[22] X. Ma, Y. Yin, and J. Zhan, "Characterizations of $h$-intra- and h-quasi-hemiregular hemirings," Computers and Mathematics with Applications, vol. 63, no. 4, pp. 783-793, 2012. 
[23] X. Ma and J. Zhan, "Generalized fuzzy $h$-bi-ideals and $h$-quasiideals of hemirings," Information Sciences, vol. 179, no. 9, pp. 1249-1268, 2009.

[24] X. Ma and J. Zhan, "New fuzzy $h$-ideals in hemirings," Politehnica University of Bucharest Scientific Bulletin A, vol. 74, no. 1, pp. 11-24, 2012.

[25] J. Zhan and Y. Yin, "A new view of fuzzy $k$-ideals of hemirings," Journal of Intelligent and Fuzzy Systems, vol. 23, no. 5, pp. 169176, 2012.

[26] B. Yu and J. Zhan, "Falling fuzzy ideals of hemirings," Journal of Intelligent and Fuzzy Systems. In press. 


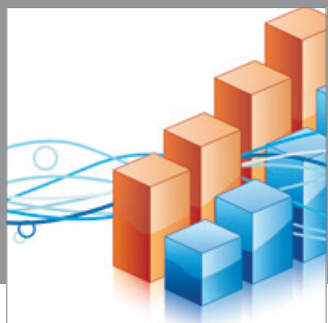

Advances in

Operations Research

mansans

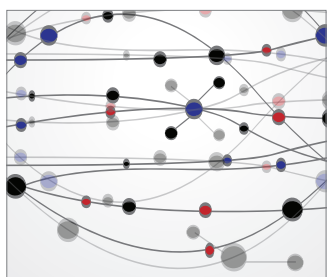

The Scientific World Journal
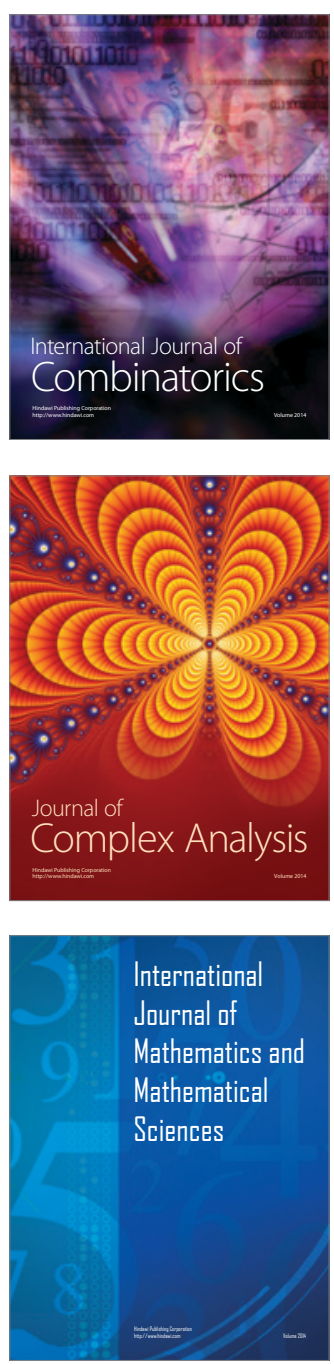
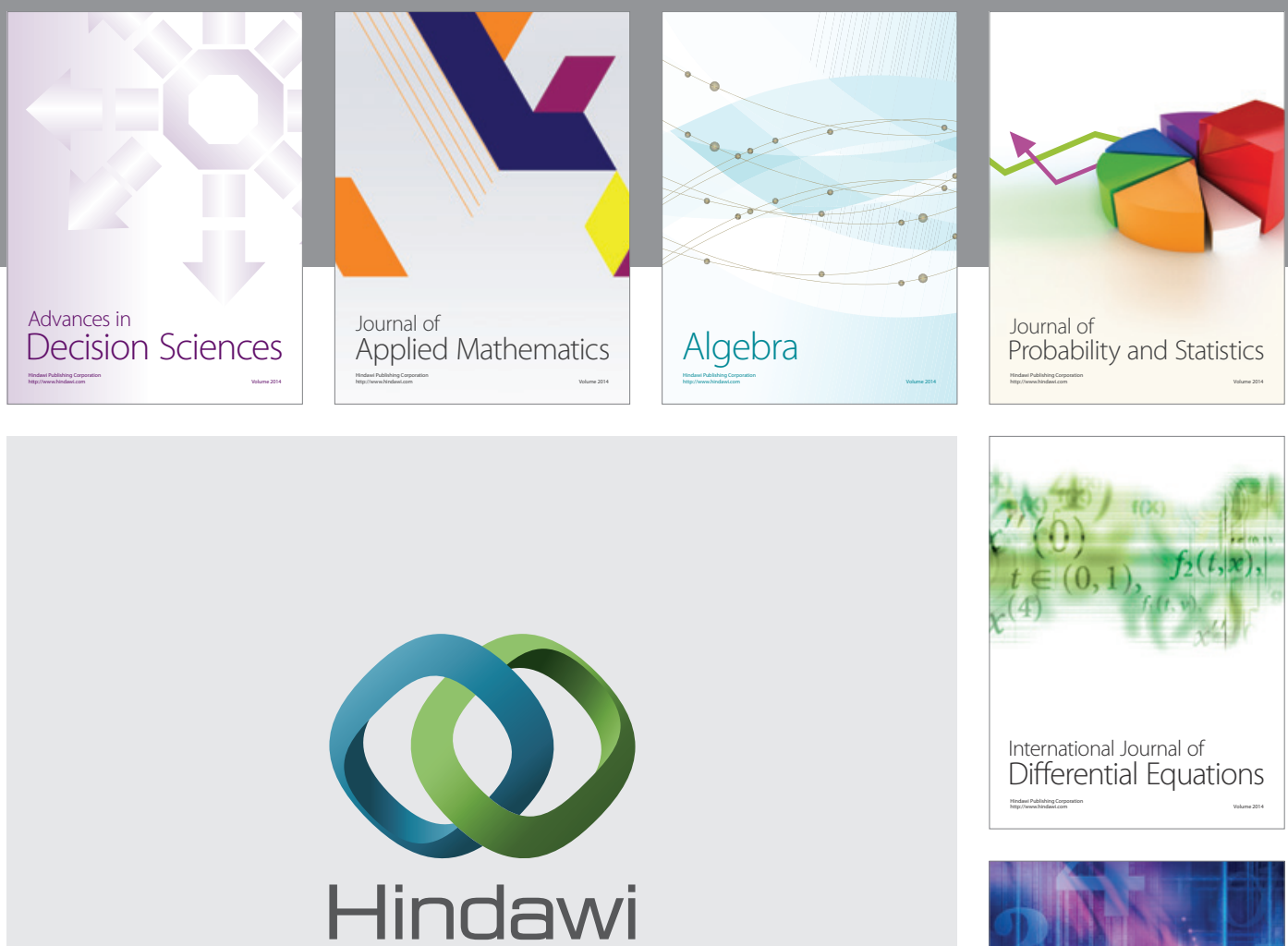

Submit your manuscripts at http://www.hindawi.com
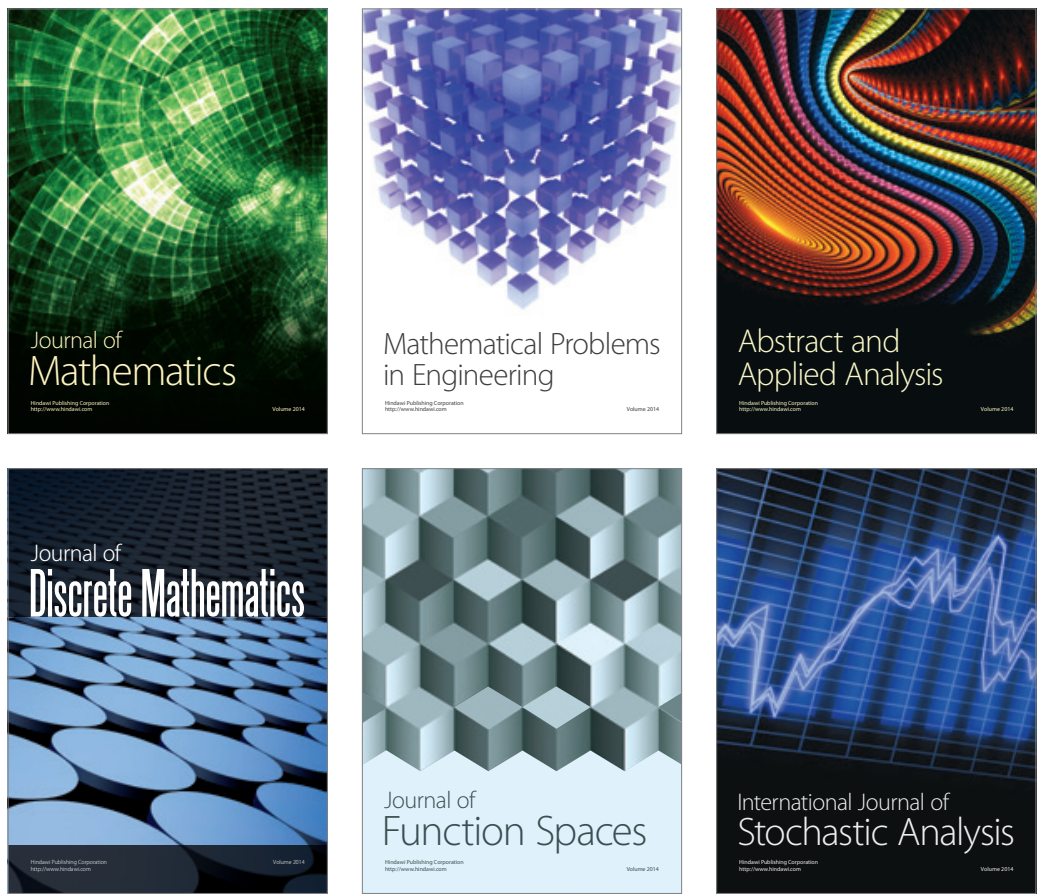

Journal of

Function Spaces

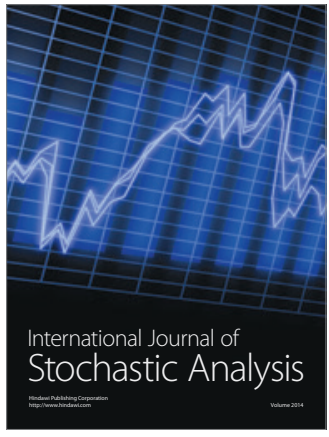

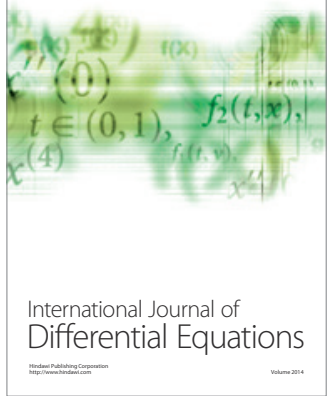
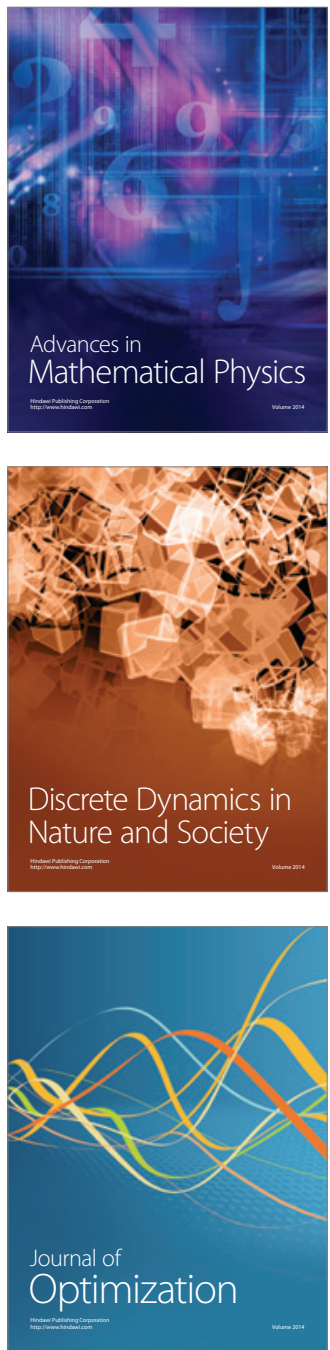